\title{
SafeWatch: Detectando quedas com Smartwatches
}

\author{
SafeWatch: Detecting falls with Smartwatches
}

\author{
Victor de Souza Tavares \\ Universidade Federal da Bahia \\ Rua Barão de Jeremoabo, s/n \\ - Ondina, 40170-115 \\ Salvador - BA, Brasil \\ victtavares1@gmail.com
}

\author{
Igor Lopes de Faria \\ Universidade Federal da Bahia \\ Rua Barão de Jeremoabo, $s / n$ \\ - Ondina, 40170-115 \\ Salvador - BA, Brasil \\ igor.faria@ufba.br
}

\author{
Vaninha Vieira \\ Universidade Federal da Bahia \\ Rua Barão de Jeremoabo, s/n \\ - Ondina, 40170-115 \\ Salvador - BA, Brasil \\ vaninha.vieira@dcc.ufba.br
}

\begin{abstract}
RESUMO
Quedas podem ter sérias consequências, a ponto de serem consideradas um grave problema de saúde pública que afeta principalmente a população idosa, onde está relacionado com a perda de confiança, autoestima, e autonomia. Esse problema se mostra ainda mais relevante se consideramos o crescente número de idosos que em busca de sua independência e autonomia decidem morar sozinhos. É crucial que o idoso tenha rápido acesso ao atendimento médico, parte importante para a sua rápida recuperação. A demora no atendimento médico está ligado ao aumento de taxas de mortalidade e gravidade em um evento de queda. Pensando nisso, foi desenvolvido o SafeWatch, um sistema de detecção de quedas embarcado em relógios inteligentes (em inglês, Smartwatch). O sistema proposto irá monitorar o idoso através de sensores presentes no smartwatch, e ao detectar uma queda, além de vibrar no pulso do usuário, irá informar para uma lista de contatos de emergência do usuário a sua localização e a possibilidade do idoso estar em uma situação de perigo. Experimentos foram realizados com oito indivíduos de biotipos distintos, onde cada um deles deveria simular uma queda em sentidos distintos. Através deste experimento, foi possível detectar o grau de confiabilidade da aplicação utilizando os valores de Sensibilidade e Especificidade que atingiram $89.06 \%$ e $100 \%$ respectivamente.
\end{abstract}

\section{Palavras-Chave}

Relógios Inteligentes, Computação Ubíqua, Detecção de quedas, Idoso.

\begin{abstract}
Falling can have serious consequences, enough to be considered a serious public health problem that affects mainly the older population, in which is related to the loss of confidence, self-esteem and autonomy.This problem is shown even more relevant if we consider the growing number of seniors, who in the search of their independence and autonomy decide to
\end{abstract}

Permission to make digital or hard copies of all or part of this work for personal or classroom use is granted without fee provided that copies are not made or distributed for profit or commercial advantage and that copies bear this notice and the full citation on the first page. To copy otherwise, or republish, to post on servers or to redistribute to lists, requires prior specific permission and/or a fee.

SBSI 2017 June $5^{\text {th }}-8^{\text {th }}$, 2017, Lavras, Minas Gerais, Brazil

Copyright SBC 2017. live alone. It is crucial that the elderly have quick access to medical care, a key part for quick recovery. The delay in medical care is linked with the increase of mortality rates and severity in a fall event. Thinking about it, the SafeWatch was developed as a fall detection system embedded in smartwatches. The proposed system will monitor the seniors through sensors at the smartwatch, and when a fall is detected, It will vibrate on the user?s wrist and report to a list of emergency contacts their location and inform the possibility of the elderly to be in a dangerous situation. Experiments were performed with eight individuals of different biotypes, in which each one of them simulated a fall event in different directions. According to the experiments, it was possible to evaluate the application reliability through the values of Sensitivity and Specificity that reached $89,06 \%$ and $100 \%$, respectively.

\section{CCS Concepts}

-Applied computing $\rightarrow$ Life and medical sciences; Health care information systems;

\section{Keywords}

Smartwatch, Ubiquitous Computing, Fall detection, Elderly

\section{INTRODUÇÃO}

Devido a diversos avanços tecnológicos e médicos, a população mundial vem envelhecendo de forma gradual. Projeções feitas pelas Nações Unidas [9], indicam que 11,57\% da população mundial tem 60 anos ou mais. Este mesmo relatório aponta que em 2050 a porcentagem de idosos irá quase dobrar, correspondendo a $21,1 \%$ da população. Esta tendência não é muito diferente no Brasil, onde de acordo com as projeções do IBGE $^{1}, 8,17 \%$ da população irá ter 65 anos ou mais em 2016, com este número aumentando para $13,44 \%$ em 2030 .

Além de ser uma parcela da população que cresce, o número de idosos que mora sozinhos também vem aumentando. De acordo com o IBGE ${ }^{2}$, entre 1992 e 2012, este número triplicou no Brasil, passando de 1,1 milhão para 3,7 milhões, um aumento de $215 \%$. Na busca pela sua independência, o idoso fica vulnerável a um dos principais problemas desta faixa etária, as quedas. De acordo com um estudo da Organização Mundial de Saúde, de $28 \%$ a $35 \%$ da população

\footnotetext{
${ }^{1}$ http://www.ibge.gov.br/apps/populacao/projecao

${ }^{2}$ http://www.ibge.gov.br/home/estatistica/populacao
} 
maior do que 64 anos sofre pelo menos uma queda por ano. De acordo com o Portal Brasil ${ }^{3}$, o SUS (Sistema Unico de Saúde) registra a cada ano, um gasto de mais de $\mathrm{R} \$ 51$ milhões com o tratamento de fraturas decorrentes de queda.

Outra questão que torna as quedas ainda mais prejudiciais à saúde física e mental do idoso é o tempo entre a queda e o atendimento médico. De acordo com Stephen R. Lord [10], a demora no atendimento está fortemente ligada ao índice de mortalidade e gravidade do acidente depois de uma queda. Na ocorrência do "long-lie", ou seja, o idoso permanece mais de uma hora no chão, metade dos idosos chegam a falecer antes dos 6 meses do ocorrido [11].

Visando minimizar essas graves consequências das quedas, diversos sistemas de detecção foram desenvolvidos nos últimos anos, porém estes sistemas não fazem uso de tecnologias mais popularizadas no mercado, ou utilizam plataformas que não são vestíveis, prejudicando a mobilidade do usuário. Por exemplo, diversos sistemas utilizam o smartphone como principal plataforma na detecção de quedas. Analisando somente as questões de popularidade e hardware, o smartphone se apresenta como uma solução plausível. De acordo com Gartner ${ }^{4}$, foram vendidos mais de 1 bilhão de aparelhos somente em 2014. Na perspectiva de hardware, a maioria dos smartphones modernos possuem giroscópio ou acelerômetro, dois dos principais sensores utilizados no reconhecimento de atividades atualmente.

Entretanto, quando pensamos em mobilidade, o smartphone passa a ser uma solução com baixo potencial, pois para que os sistemas funcionem corretamente, os mesmos precisam estar fixos em uma posição pré-estabelecida, como o bolso ou pulso do usuário [12]. Isso faz com que, em situações corriqueiras, como colocar o celular na bolsa, possa gerar um número grande de falsos positivos. Este trabalho propõe como solução a criação de um sistema de detecção de quedas através de uma solução integrada entre smartphone e o smartwatch. O smartwatch será responsável pela detecção dos eventos de queda, enquanto o smartphone será responsável pelo gerenciamento dos contatos e envio das mensagens de emergência.

O smartwatch é uma ferramenta que permite que este tipo de aplicação seja de fácil interação e invisível para o usuário, além de ter uma capacidade de processamento bastante similar aos smartphones, com uma popularidade crescente. A Samsung, umas das empresas pioneiras no mercado de smartwatches, lançou em outubro de 2015 o Samsung Gear S2. O Gear é um exemplo de como esses sistemas estão cada vez mais poderosos. Ele possui uma memória RAM de $512 \mathrm{MB}$ e $4 \mathrm{~GB}$ de armazenamento, conectividade $W i F i$ e $4 \mathrm{G}$ além de diversos sensores como giroscópio e acelerômetro Samsung ${ }^{5}$. A popularidade desta plataforma é vista através do número de smartwatches vendidos. No ano de 2015, 30,32 milhões de aparelhos foram vendidos, e a previsão é de que, em 2016, este número suba para 50,40 milhões.

Este trabalho ter por objetivo desenvolver um sistema de detecção de quedas utilizando os smartwatches já popularizados no mercado como ferramenta principal no desenvolvimento desta solução. O sistema deverá exigir o mínimo de interação possí- vel do usuário, sendo capaz de detectar as quedas de maneira automática. Também é dese-

\footnotetext{
${ }^{3}$ http://www.brasil.gov.br/saude/2012/04/quedas

${ }^{4}$ http://www.gartner.com/newsroom/id/2996817/

${ }^{5}$ http://www.samsung.com/global/galaxy/gear-s2/spec/
}

jável que o sistema utilize o mínimo de sensores possível, visando diminuir o custo computacional da solução apresentada, mantendo a acurácia semelhante aos principais sistemas de detecção presentes na literatura.

Para realizar este trabalho a seguinte metodologia foi adotada: inicialmente investigamos os sistemas de detecção de quedas existentes na literatura, como foco nos algoritmos de detecção utilizados. Em seguida desenvolvemos o SafeWatch, utilizando um algoritmo de detecção de quedas que utiliza somente os dados do acelerômetro como entrada. Por fim, elaboramos um experimento para avaliar a solução proposta.

Participaram do nosso experimento oito pessoas, sendo três homens e cinco mulheres. Simulamos quatro tipos de queda e quatro tipos de atividades diárias. Os resultados indicaram que o sistema possui uma acurácia de 94,17\%.

As seguintes seções estão organizadas da seguinte maneira: A Seção 2 apresenta os trabalhos correlatos; A seção 3 apresenta o SafeWatch, o sistema de Detecção de Quedas desenvolvido através de uma solução integrada entre o smartphone e o smartwatch. A seção 4 apresenta o experimento realizado, e realiza a avaliação da ferramenta. Por fim, na seção 5 , seguem as conclusões e considerações finais.

\section{TRABALHOS CORRELATOS}

De forma geral, podemos definir uma queda como um evento súbito e involuntário, onde o indivíduo de uma posição em pé ou sentado, passa a ocupar uma posição integral ou parcialmente deitada [6]. Uma outra definição mais formal de queda descreveu este evento como "Vir ao chão ou algum nível mais baixo, sem a intenção, como consequência de um golpe violento, perda de consciência, ou início súbito de paralisia, como no caso de um acidente vascular cerebral ou um ataque epiléptico" [4].

Não existe na literatura um algoritmo ou dispositivo padrão para o desenvolvimento de SDQ(Sistema de Detecção de Queda). A plataforma vestível é bastante promissora por estar naturalmente aclopada a alguma parte do corpo do usuário, sendo possivel criar um algoritmo de detecção mais confiável, já que, como visto em Casilari [2], grande parte dos algoritmos de detecção de quedas tem como pré-condição para o seu bom funcionamento que o dispositivo esteja localizado em uma posição fixa do corpo, como cintura, pulso ou cabeça Serão discutidos três sistemas de detecção de quedas embarcardos em plataformas vestíveis. Estes três sistemas foram escolhidos pela semelhança dos mesmos com a solução proposta neste trabalho.

O primeiro destes sistemas é o SPEEDY. Ele foi o primeiro protótipo de um relógio detector de quedas construído em um smartwatch [3]. Em seu trabalho, ele utilizou dois sensores que são capazes de medir a aceleração através de 3 eixos x, y, z. O algoritmo de detecção de quedas do Speedy utiliza um algoritmo baseado em limiares com 3 valores distintos: o valor de SMV calculado através da formula que pode ser vista em 1 e dois valores de velocidade distintos chamados de v1 e v2. O Speedy foi avaliado através de quedas simuladas por 3 indivíduos em um colchão. Cada indivíduo simulou quedas em 3 posições diferentes: Frente, lado e costas. Foram realizadas um total de 45 quedas, onde $65 \%$ delas foram corretamente marcadas como um evento de queda. 


$$
S M V=\sqrt{X_{i}^{2}+Y_{i}^{2}+Z_{i}^{2}}
$$

Um outro sistema SDQ foi o F2D, uma applicação Android embarcada em um smartwatch AW-420.RX da Simvalley Mobile2 [7]. O algoritmo implementado no F2D tem como entrada os dados do acelerômetro, levando em consideração os movimentos realizados depois de um evento de queda e a localização do usuário. Para que se possa detectar as quedas, o F2D utiliza um algoritmo baseado em limiares, onde os limiares foram definidos utilizando um banco de dados com mais de 150 eventos simulados de queda.

Por fim, temos o sistema proposto por Hsieh et al [5] que utiliza dois dispositivos vestíveis acoplados no pulso do usuário. Cada um dos dispositivos está equipado com um módulo Zigbee3 responsável pela transmissão dos dados e um acelerômetro e giroscópio de 3 eixos. A frequência tanto do acelerômetro quanto do giroscópio foram configuradas para $50 \mathrm{~Hz}$, ou seja, os dados são coletados a cada $20 \mathrm{~ms}$. O algoritmo proposto utiliza ambos os dados do acelerômetro e do giroscópio para realizar a detecção de quedas. Os dados do giróscopio funcionam como um filtro inicial, desconsiderando a maioria das ativididades cotidianas, enquanto os dados do acelerômetro são responsáveis por realizar o julgamento final. O algoritmo utilizado, assim como os demais, é baseado em limiares onde os limiares foram definidos atravês de um treinamento inicial.

Cada um dos sistemas SDQ apresentam suas particularidades, apesar de todos utilizarem tecnologias vestíveis para realizarem a detecção das quedas. O Speedy foi desenvolvido utilizando um dispositivo próprio, fazendo com que os usuários precisem adquirir um dispositivo vestível que irá realizar exclusivamente a detecção de quedas fazendo com que a sua adesão seja mais complexa do que em um sistema que já utilize smartwatches já consolidados no mercado. Enquanto isso, O F2D o utiliza dados do contexto para definir se o evento ocorrido foi potencialmente perigoso ou não, o que o diferencia da maioria das soluções presentes na literatura. Já o sistema proposto por Hsieh et al [5] possui um algoritmo capaz de diferenciar $\mathrm{AD}$, como bater palmas e deitar-se, de um evento de queda em 95\% dos casos. No entanto para possuir essa acurácia, é necessário a utilização de 2 dispositivos, o que pode torna-se desconfortável para o usuário.

\section{SAFEWATCH}

Neste trabalho, apresentamos o SafeWatch, uma solução integrada entre smartphone e smartwatch, onde quedas são detectadas de maneira automatizada através de um algoritmo de detecção baseado em limiares. Quando necessário, os contatos de emergência do idoso são informados de sua localização para que possa prestar socorro de forma mais rápida possível. As seções desse capítulo são organizadas da seguinte maneira: A Seção 3.1 mostra a arquitetura que foi definida e utilizada pela ferramenta construída; A Seção 3.2 descreve detalhes da implementação do SafeWatch.

\subsection{Arquitetura}

O SafeWatch foi desenvolvido para funcionar como um aplicativo Android Wear para smartwatches que funciona em conjunto com o smartphone Android do usuário, através de uma aplicação homônima, que está sincronizada com o mesmo.
De forma geral, a aplicação smartwatch irá monitorar as atividades do usuário através do acelerômetro e no momento em que uma queda for detectada emitirá um alerta vibratório juntamente com um sinal para o smartphone. No smartphone está presente uma aplicação de gerenciamento geral do sistema, onde o usuário é capaz de adicionar, visualizar e remover os contatos de emergência que seriam notificados no momento de uma queda. Na ocorrência de uma queda o sistema irá se comportar como pode ser visto na 1.

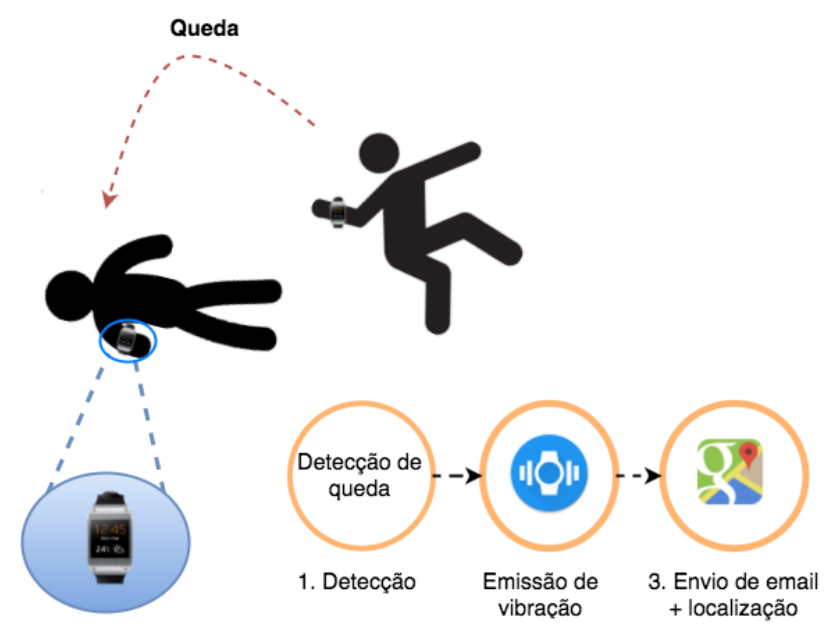

Figure 1: Aplicação no evento de queda. Figura Elaborada pelo autor (2016).

Assim que um evento de queda é detectado, o smartwatch irá emitir um sinal de vibração por um tempo determinado, e caso o usuário não informe o contrário, um email contendo a localização do usuário que está utilizando o smartwatch é enviado para os seus contatos de emergência previamente cadastrados.

O SafeWatch foi desenvolvido com base em uma arquitetura pré-definida e possui os seus módulos desacoplados para facilitar futuras mudanças ou melhorias. O fluxo de dados do SafeWatch começa com a obtenção dos dados do acelerômetro e termina com envio da mensagem de emergência. Os módulos presentes nesta aplicação são os seguintes:

1. Sensor Reader: Responsável pela configuração e gerenciamento dos sensores.

2. Fall Detector: Recebe informações provindas do Sensor Reader para através do algoritmo de detecção de quedas categorizar um determinado evento como queda ou não.

3. Watch Communicator: Realiza a comunicação entre o smartwatch e o smartphone do usuário. Irá receber dados do smartwatch que são tratados pelo módulo chamado de Fall Handler.

4. Fall Handler: Será responsável pelas ações do smartphone após um evento de queda, como o envio de emails e o gerenciamento dos dados do acelerômetro recebidos do smartwatch.

5. Contact Manager: Será responsável pelo gerenciamento dos contatos de emergência do usuário. Ações 
como visualização, adição e remoção de contatos estão encapsuladas neste módulo.

Os módulos Sensor Reader e Fall Detector estão presentes na aplicação embarcada no smartwatch, os demais módulos estão presentes na aplicação para smartphones

\subsection{Implementação}

A implementação do SafeWatch foi dividida em várias partes, onde cada uma delas é representada por um módulo independente dos demais. A linguagem de programação utilizada foi Java, linguagem padrão no desenvolvimento de aplicações Android. Nas seções a seguir são demonstrados detalhes da implementação e funcionamento de cada módulo.

\subsubsection{Sensor Reader}

O módulo Sensor Reader é responsável pela configuração e gerenciamento do acelerômetro. Aqui, o acelerômetro é configurado para atualizar seus dados a uma frequência de $50 \mathrm{~Hz}$, ou seja, a cada $20 \mathrm{~ms}$. Os dados do acelerômetro são coletados a todo momento, mesmo quando a aplicação não está em primeiro plano.

Este módulo também será responsável por armazenar os dados do acelerômetro nos últimos 0.4 segundos para posterior uso no algoritmo de detecção, caso necessário. Tanto a escolha da frequência de $50 \mathrm{~Hz}$ quanto o tempo de 0.4 segundos para o armazenamento de dados do acelerômetro serão explicados com mais detalhes na Seção 3.3.2.

\subsubsection{Fall Detector}

O módulo Fall Detector encapsula o algoritmo de detecção de quedas baseado em limiares utilizado pelo SafeWatch. Este é o módulo mais complexo da aplicação, pois nele se encontra a lógica responsável por decidir, através dos dados obtidos do acelerômetro, se um evento de queda ocorreu ou não. $\mathrm{O}$ algoritmo proposto é uma adaptação do algoritmo desenvolvido por [5]. O algoritmo proposto neste trabalho se diferencia do algoritmo proposto em Hsieh et al.[5] pela não utilização do giroscópio como sensor auxiliar, pois acredita-se que sem o uso desse sensor é possível obter-se resultados satisfatórios. O giroscópio é usado por Hsieh et al. (2014) para aumentar o valor de Especificidade do sistema proposto. Ele é utilizado como o primeiro passo no algoritmo baseado em limiares utilizado por ele. Nos experimentos realizados por Hsieh et al. (2014), a velocidade angular obtida através do giroscópio possui valores maiores que $350 \check{z} / \mathrm{s}$ em um evento de queda.

De acordo com um estudo feito por Casilari[2], o número de sensores utilizados afeta diretamente a duração da bateria. Em um experimento realizado por Mellone et al. [8] utilizando um smartphone Samsung Galaxy S II, a duração da bateria aumentou de 16 para 30 horas ao utilizar somente um sensor (acelerômetro) em vez de três sensores (acelerômetro, magnetômetro, giroscópio). Além disso, o SafeWatch só utiliza um smartwatch, enquanto o sistema proposto por Hsieh et al. [5] necessita de dois dispositivos de pulso similares a um smartwatch.

Um grande desafio quando utilizamos um algoritmo baseado em limiares é a definição dos valores dos limiares. Caso esse valor seja muito alto, o sistema irá deixar escapar alguns eventos de queda, mas não irá categorizar uma $\mathrm{AD}$ como uma queda. Do outro lado, se esse valor for muito baixo, o sistema irá detectar todos os eventos de queda, mas algumas $\mathrm{AD}$ pode ser categorizadas como eventos de queda de maneira equivocada. De acordo com o treinamento inicial realizado por Hsieh et al. [5], o valor de SMV, representado pela fórmula 1 , será maior que $6 \mathrm{G}$, onde $\mathrm{G}=9.8 \mathrm{~m} / \mathrm{s}$, no momento do impacto em um evento de queda

Também foi identificado por Hsieh et al. [5], que caso o valor de aceleração atinja o valor de $6 \mathrm{G}$, o valor do desvio padrão ficava com valores em torno de $1.07 \mathrm{G}$ em movimentos regulares do braço realizados 0.4 segundos antes e depois deste pico de Também foi identificado por Hsieh et al. (2014), que caso o valor de aceleração atinja o valor de $6 \mathrm{G}$, o valor do desvio padrão ficava com valores em torno de $1.07 \mathrm{G}$ em movimentos regulares do braço realizados 0.4 segundos antes e depois deste pico de aceleração.Entretanto, em eventos de queda, este valor estava mais próximo de 1.69G.

Por fim, o período de inatividade posterior a uma queda foi analisado. De acordo com Hsieh et al. [5], o valor da Soma das Acelerações (SMA), expresso através da Equação 2 , tem uma relação diretamente proporcional com o nível de movimentação de um corpo. Foi identificado que em eventos de queda, o indíviduo tende a ficar parado por pelo menos 2 segundos, com valores de SMA inferiores a 200G. É importante ressaltar que este valor de $200 \mathrm{G}$ é encontrado quando a frequência do acelerômetro é de $50 \mathrm{~Hz}$, ou seja, a cada $20 \mathrm{~ms}$ um novo valor do acelerômetro é coletado. Caso está frequencia seja diferente, o número de amostra coletados será afetado, fazendo com que o valor de SMA seja diferente de 200G em um periodo de inatividade.

$$
S M A=\sum_{i=1}^{N}\left(\left|X_{i}\right|+\left|Y_{i}\right|+\left|Z i_{i}\right|\right)
$$

Nesta equação Xi, Yi, Zi, são os valores da aceleração no tempo i e $\mathrm{N}$ é o número de amostras desejadas. Levando como base o treinamento inicial descrito acima foi possível desenvolver o algoritmo descrito na Figura 2.

1. Os valores do acelerômetro são monitorados, caso SMV seja maior do que 6G,os demais valores de SMV são monitorados por mais 0.4 segundos. O maior dosvalores observado neste tempo é marcado como pico de aceleração e o algoritmo prossegue para o passo 2. 2. O desvio padrão de SMV é calculado, nos 0.4 segundos anteriores e posteriores a detecção do maior valor de SMV. Caso este valor não seja menor do que $1.5 \mathrm{G}$, o algoritmo irá para o passo 3. 3. O valor de SMA é calculado, e caso este valor seja menor do que $200 \mathrm{G}$ finalmente confirmamos que um evento de queda ocorreu.

Na figura 3, é possível ver os momento em que o sistema realiza a leitura de dados do acelerômetro, demonstrado pela tela da esquerda. Esta leitura é feita de maneira constante, mas não afeta o funcionamento das demais funções do smartwatch.

\subsubsection{Watch Communicator}

O módulo Watch Communicator é responsável pela comunicação entre o smartwatch e o smartphone do usuário. Fisicamente, esta comunicação é realizada via bluetooth, já a nível de software, está comunicação é realizada através do que chamamos na arquitetura Android de Services.

No Android, um Service é um componente da aplicação capaz de realizar operações de longa duração em segundo 


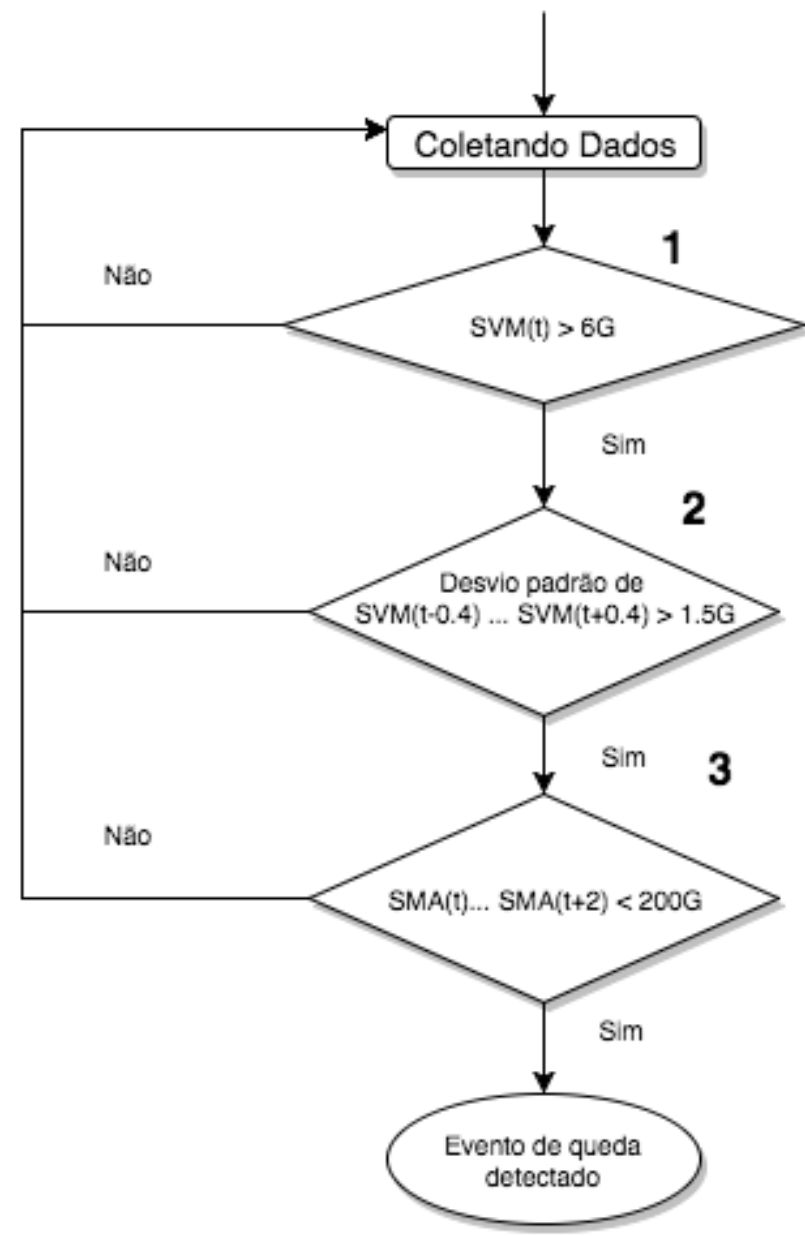

Figure 2: Fluxograma do algoritmo proposto. Figura Elaborada pelo autor (2016).

plano ${ }^{6}$. No SafeWatch, os Services recebem os dados do acelerômetro referentes ao evento de queda, ou seja, todos os registros do acelerômetro 0.4 segundos antes do pico de aceleração, até 2 segundos depois deste valor. Além disso, também é enviado, uma variável booleana indicando se deve-se ou não enviar um e-mail para a lista de contatos de emergência do usuário. Os emails de emergência são enviados para a lista de contato do usuário 15 segundos após um evento de queda, ou antes disso, caso o usuário confirme que precisa de ajuda.

Para que os contatos da lista de emergência não sejam incomodados desnecessariamente na ocorrência de falsas detecções de quedas, o usuário poderá cancelar o envio dos emails de emergência, dentro de 15 segundos após um evento de queda, caso ele informe que está bem. O tempo de 15 segundos foi escolhido, pois o usuário terá tempo de dar um feedback para o sistema, caso ele não esteja em uma situação de perigo. Da mesma forma, este tempo não irá atrasar o envio da mensagem de emergência quando o usuário realmente precisar.

\subsubsection{Fall Handler}

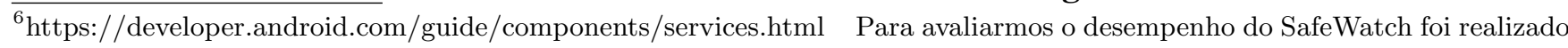

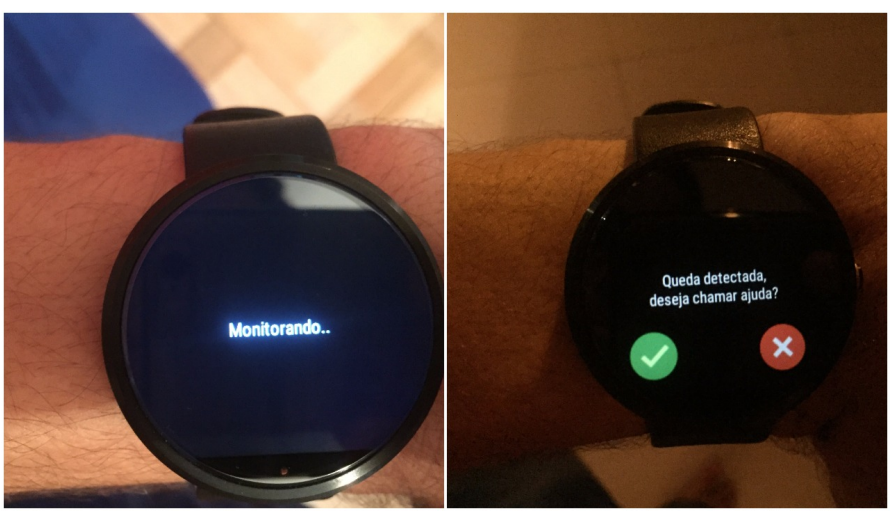

Figure 3: Na ordem, telas de monitoramento e queda detectada. Figura Elaborada pelo autor (2016).

Este módulo é responsável pelo envio de e-mails e a manipulação de arquivos com os dados de uma queda. Para que se possa realizar o envio de e-mails, foi criado um email padrão do SafeWatch. O envio de e-mail é feito de forma assíncrona, sem bloquear a interação do usuário com a aplicação. O modelo do email, além de enviar uma mensagem informando que o usuário pode está em uma situação de perigo, um link do Google Maps é anexado com a última localização do usuário obtida pelo sistema.

Os dados referentes a um evento de queda são salvos na raiz do sistema de arquivos do smartphone android na pasta /SafeWatch/smartwatch. O arquivo é nomeado com o padrão experimentData, onde timeStamp representa o momento do salvamento do arquivo, em milisegundos. O arquivo está salvo no formato CSV, com os valores de aceleração nos eixos $\mathrm{x}, \mathrm{y}, \mathrm{z}$, o valor de SMV correspondente ao registro e o tempo em milisegundos em que ele ocorreu. Apesar destes valores não serem úteis para o usuário final, eles poderão servir para uma possível análise dos dados de queda e replica dos experimentos realizados.

\subsubsection{Contact Manager}

Neste módulo, estão encapsuladas as ações de adição, remoção e listagem dos contatos de emergência do usuário. O cadastro dos contatos de emergência deve ser a primeira ação do usuário no primeiro contato com a aplicação. Depois de adicionar os contatos de emergência, o usuário não necessita realizar mais nenhum tipo de cadastro ou configuração no sistema.

\section{EXPERIMENTO}

Nesta seção será apresentado o processo de avaliação utilizado para verificar a precisão do sistema de detecção de quedas proposto. As seções desse capítulo são organizadas da seguinte maneira: A Seção 4.1 apresenta os detalhes da metodologia utilizada para avaliar o SafeWatch; A Seção 4.2 mostra as métricas utilizadas na avaliação; A Seção 4.3 apresenta os resultados obtidos no experimento realizado e faz uma comparação com os resultados obtidos em outros trabalhos.

\subsection{Metodologia}


uma série de experimentos. $\mathrm{O}$ algoritmo de detecção de quedas proposto foi avaliado através de um conjunto de quedas simuladas e também um conjunto de atividades diárias realizadas pelos participantes do experimento. O grupo de voluntários possui um perfil diversificado, sendo composto de 3 homens e 5 mulheres. O experimento não foi realizado com nenhum idoso devido a grande dificuldade de simular eventos de queda sem por em risco a integridade física do mesmo. Além disso, as bases de dados encontradas referentes a eventos de quedas com idosos são privadas e não foram disponibilizadas, como vista no trabalho proposto por Kostopoulos et al. [7].

O smartwatch utilizado para realizar o experimento foi um Moto 360 da 1 ž geração ${ }^{7}$.

Para avaliar o algoritmo de detecção de quedas implementado, foi realizado o seguinte experimento, composto de três etapas:

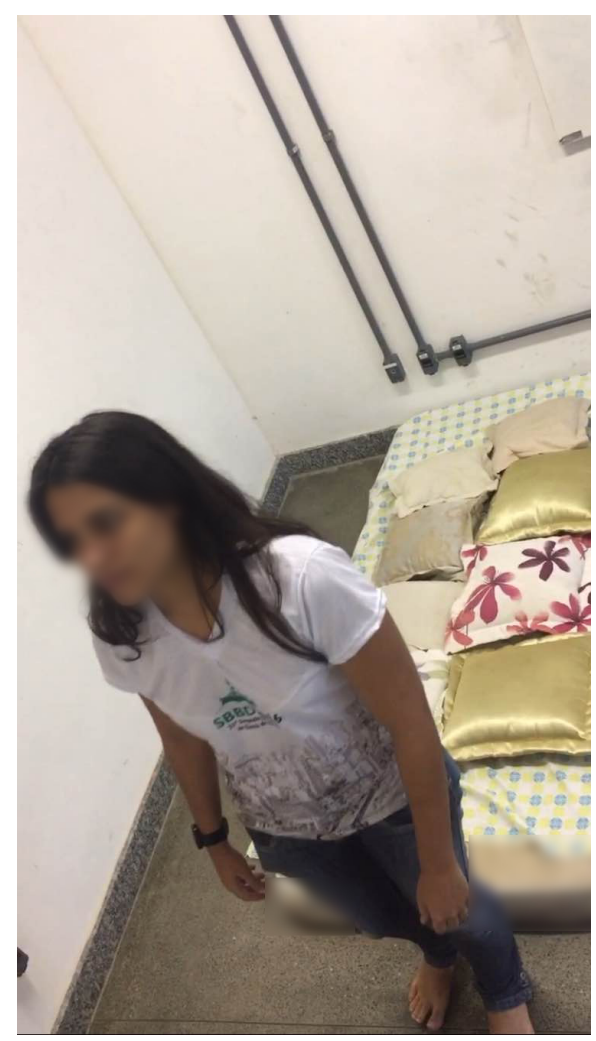

Figure 4: Usuário em preparação para uma queda de costas. Figura Elaborada pelo autor (2016).

- Preparação: Nesta etapa é solicitado que o usuário coloque o smartwatch em seu pulso e ajuste a pulseira do relógio de uma maneira que o smartwatch permaneça firme, mas confortável. Depois disso, é informado que o usuário deverá simular duas quedas em cada um dos sentidos escolhidos: Costas, Frontal, Lado Direito, Lado Esquerdo. A ordem das quedas é decidida pelo usuário, o único requisito é que ele realize todas as oito quedas.

- Realização das Quedas: O usuário irá se posicionar de pé, na frente de um colchão coberto de almofadas como

\footnotetext{
$\overline{{ }^{7} \text { https://www.motorola.com/us/products/moto-360 }}$
}

pode ser visto na figura 4 . A partir desta posição, ele irá realizar as oitos quedas, duas de cada tipo, como descrito na etapa anterior.A ordem das quedas é escolhida pelo usuário.

- Realização de Atividades Diárias: Nesta etapa solicitamos que o usuário realize 4 atividades do seu cotidiano. Elas são: sentar em uma cadeira, levantar de uma cadeira, deitar no colchão e levantar do colchão. Estas atividades são realizadas afim de verificar se o sistema proposto é capaz de distinguir atividades diárias de um evento de queda. O sistema proposto não é capaz de distinguir qual a atividade diária que está sendo realizada, ele só é capaz de diferencia-la de um evento de queda.

Além de executarem as simulações de queda antes das atividades diárias, ambos os eventos foram executados de maneira isolada, ou seja, não foi seguida nenhuma ordem ou sequência pré-definida de eventos

\subsection{Métricas de Avaliação}

Para que pudessemos analisar a performance do sistema de detecção de quedas foram utilizadas três métricas, a Sensibilidade, Especificidade e Acurácia. De acordo com Casilari [1], os valores de Sensibilidade e Especificidade são duas métricas bastante utilizadas na literatura para a análise de performance em sistemas de detecção de quedas. Elas representam, respectivamente, a proporção de eventos de queda e AD que foram classificadas corretamente como tal. Já a Acurácia é uma combinação da Sensibilidade e da Especificidade e nos dá uma ideia geral da performance do sistema. A Sensibilidade é expressa pela fórmula 3 . As variáveis TP e FN são, respectivamente, acrónimos para True Positive (Verdadeiro Positivo em inglês) e False Negative (Falso Negativo em inglês). A variável TP representa o número de eventos de queda corretamente classificadas, enquanto $\mathrm{FN}$ representa as quedas que não foram detectadas pelo sistema.

$$
\text { Sensibilidade }=\frac{T P}{T P+F N}
$$

Já a Especificidade é expressa pela fórmula 4. As variáveis TN e FP são, respectivamente acrónimos para True Negative (Verdadeiro Negativo em inglês) e False Positive (Falso Positivo em inglês). A variável TN representa o número de atividades diárias corretamente classificadas como tal, enquanto FP representa as atividades diárias que foram classificadas como queda.

$$
\text { Especificidade }=\frac{T N}{F P+T N}
$$

\subsection{Resultados}

Na tabela 1 podemos ver os resultados dos experimentos de queda para cada um dos indivíduos. Como descrito na Seção 4.1 cada individuo realizou duas quedas em quatro sentidos diferentes. Cada elemento da tabela representa o número de quedas que foram identificadas com sucesso pelo sistema em cada uma das direções.

Como podemos ver na tabela 1 , o número de verdadeirospositivos é de 57, enquanto o número de falsos-negativos é de somente 7 , o que nos dá uma especificidade de 89,06\%. Foi possível observar que o limiar inicial de $6 \mathrm{G}$ no algoritmo 
Table 1: Resultados do experimentos de Queda.

\begin{tabular}{l|c|c|c|c}
\hline Individuo & Frente & Costas & Direita & Esquerda \\
Individuo 1 & 2 & 1 & 2 & 2 \\
Individuo 2 & 1 & 2 & 2 & 2 \\
Individuo 3 & 2 & 2 & 1 & 2 \\
Individuo 4 & 2 & 2 & 2 & 2 \\
Individuo 5 & 2 & 1 & 2 & 1 \\
Individuo 6 & 2 & 2 & 2 & 1 \\
Individuo 7 & 2 & 2 & 2 & 2 \\
Individuo 8 & 2 & 1 & 2 & 2
\end{tabular}

de detecção de quedas não foi alcançado em todos os eventos erroneamente não categorizados como queda.

Já na tabela 2, podemos ver os resultados do experimentos de AD para cada um dos indivíduos. Cada um deles realizou quatro tipos de AD, como descrito na Seção 4.1. Cada elemento da tabela representa o número de $\mathrm{AD}$ que não foram identificadas pelo sistema como um evento de queda.

Table 2: Resultados do experimentos de Atividades Diárias.

\begin{tabular}{c|c|c|c|c}
\hline Individuo & Levantar & Sentar & Levantar & Deitar \\
Individuo 1 & 2 & 2 & 2 & 2 \\
Individuo 2 & 2 & 2 & 2 & 2 \\
Individuo 3 & 2 & 2 & 2 & 2 \\
Individuo 4 & 2 & 2 & 2 & 2 \\
Individuo 5 & 2 & 2 & 2 & 2 \\
Individuo 6 & 2 & 2 & 2 & 2 \\
Individuo 7 & 2 & 2 & 2 & 2 \\
Individuo 8 & 2 & 2 & 2 & 2
\end{tabular}

Como visto na Tabela 3, o algoritmo proposto neste trabalho apresentou resultados satisfatórios identificando um evento de queda em quase $90 \%$ dos casos, e não apresentando nenhum falso-positivo nas $\mathrm{AD}$ testadas. Em comparação com o Speedy, sistema desenvolvido por [3], o nosso sistema apresentou uma sensibilidade $24,06 \%$ maior, ou seja, o SafeWatch apresentou uma especificidade de 89,06\% contra $65 \%$ do Speedy em experimentos bastantes similares.

Table 3: Comparação com os resultados dos Trabalhos Relacionados.

\begin{tabular}{c|c|c|c|c} 
Sensibilidade & SafeWatch & Speedy & F2D & FDS \\
Especificidade & $89,06 \%$ & $65 \%$ & $93,48 \%$ & $95 \%$ \\
Acurácia & $100 \%$ & - & $98,54 \%$ & $96,07 \%$ \\
& $94,53 \%$ & - & $96,01 \%$ & $95,85 \%$
\end{tabular}

Entretanto em comparação com Hsieh et al.[5], este trabalho apresentou uma sensibilidade 5,94\% menor e uma especificidade $3,3 \%$ maior. Um dos prováveis motivos desse valor menor de Sensibilidade são as condições do experimento. Os experimentos realizados por Hsieh et al. [5] foram em uma superfície acolchoada mais fina que um colchão convencional, o que leva a uma aceleração maior no impacto, podendo diminuir o número de falsos-negativos no experimento realizado. Em relação ao tipos de quedas realizados, tanto este, quanto o trabalho proposto por Hsieh et al.[5] apresentaram quatro tipos de quedas: frontais, laterais (Direito e Esquerdo), Costas. Já em relação as $\mathrm{AD}$, ele apresentou um número maior de atividades, incluindo andar e correr, o que pode levar a número maior de falso-positivos

\section{CONCLUSÃO}

A proposta deste trabalho foi criar um sistema de detecção de quedas que exige-se o mínimo de interação possível do usuário, consumindo o mínimo de recursos com uma precisão similar aos demais SDQ existentes através de uma plataforma vestível que esteja se popularizando no mercado.

As maiores dificuldades encontradas foram no desenvolvimento do algoritmo de detecção de quedas. Com a proposta inicial de utilizar somente o acelerômetro, diferente de demais sistemas que também utilizam o giroscópio, a acurácia do sistema poderia ser afetada, caso algum dos limiares não se adaptassem a essa nova proposta. Outra dificuldade foi entender as características de uma queda a partir de sua aceleração, alguns conceitos físicos não são tão triviais, o que levou a muita pesquisa

Por fim, foi desenvolvido o SafeWatch, um sistema de detecção embarcado em um smartwatch que utiliza o smartphone como uma plataforma auxiliar. A interface é simples, e permite que o usuário interaja com o sistema de maneira fácil e somente quando necessário.

Utilizando somente um único sensor e um único dispositivo smartwatch, o SafeWatch apresentou resultados satisfatórios com uma acurácia similar ou melhor que outros SDQ existentes.

Para que possamos melhorar a solução proposta poderiamos analisar e buscar meios de otimizar o consumo de bateria do smartwatch mesmo com o constante monitoramento dos dados dos sensores, integrar com outros tipos de sensores, como o sensor de batimento cardíaco, a fim de aumentar ainda mais a acurácia do sistema, utilizar outros meios de comunicação de emergência além do email, realizar o teste da aplicação com um número maior de $\mathrm{AD}$, principalmente aquelas que exigem uma movimentação maior do braço do individuo, como andar ou correr e realizar testes investigando o impacto de mais tipos de quedas na acurácia do sistema, por exemplo, diferenciar quedas de um usuário consciente de um usuário inconsciente.

\section{REFERENCES}

[1] M. A. Casilari, Eduardo e Oviedo-Jiménez. Automatic fall detection system based on the combined use of a smartphone and a smartwatch. PLoS one, 10(11):e0140929, 2015.

[2] R. e. M. M.-J. Casilari, Eduardo e Luque. Analysis of android device-based solutions for fall detection. Sensors, 15(8):17827-17894, 2015.

[3] T. Degen, H. Jaeckel, and S. Rufer, Michael e Wyss. Speedy: A fall detector in a wrist watch. In $I S W C$, pages 184-189, 2003.

[4] M. J. Gibson, R. O. Andres, B. Isaacs, T. Radebaugh, and J. Wormpetersen. The prevention of falls in later life-a report of the kellogg-international-work-group on the prevention of falls by the elderly. Danish Medical Bulletin, 34:1-24, 1987.

[5] S.-L. Hsieh, C.-C. Chen, S.-H. Wu, and T.-W. Yue. A wrist-worn fall detection system using accelerometers and gyroscopes. In Networking, Sensing and Control (ICNSC), 2014 IEEE 11th International Conference on, pages 518-523. IEEE, 2014.

[6] R. Igual, C. Medrano, and I. Plaza. Challenges, issues and trends in fall detection systems. Biomedical engineering online, 12(1):1, 2013. 
[7] P. Kostopoulos, T. Nunes, K. Salvi, M. Deriaz, and J. Torrent. F2d: A fall detection system tested with real data from daily life of elderly people. In 201517 th International Conference on E-health Networking, Application 85 Services (HealthCom), pages 397-403. IEEE, 2015.

[8] S. Mellone, C. Tacconi, L. Schwickert, J. Klenk, C. Becker, and L. Chiari. Smartphone-based solutions for fall detection and prevention: the farseeing approach. Zeitschrift für Gerontologie und Geriatrie, 45(8):722-727, 2012.

[9] Na $\tilde{A} \breve{g} \tilde{A} t$ es Unidas. World population ageing 2013. Report, The Department of Economic and Social Affairs of the United Nations, 2013.

[10] C. S. e. H. B. M. Stephen R. Lord. Falls in older people: risk factors and strategies for prevention. Cambridge University Press, Cambridge, 2001.

[11] D. Wild, U. Nayak, and B. Isaacs. How dangerous are falls in old people at home? $\mathrm{Br}$ Med J (Clin Res Ed), 282(6260):266-268, 1981.

[12] S.-D. B. Yi He, Ye Li. Fall detection by built-in tri-accelerometer of smartphone. Proceedings of 2012 IEEE-EMBS International Conference on Biomedical and Health Informatics, 25:184-187, 2012. 